TRANSACTIONS OF THE

AMERICAN MATHEMATICAL SOCIETY

Volume 359, Number 9, September 2007, Pages 4389-4403

S 0002-9947(07)04222-5

Article electronically published on March 20, 2007

\title{
DERIVATIONS OF AN EFFECTIVE DIVISOR ON THE COMPLEX PROJECTIVE LINE
}

\author{
MAX WAKEFIELD AND SERGEY YUZVINSKY
}

\begin{abstract}
In this paper we consider an effective divisor on the complex projective line and associate with it the module $D$ consisting of all the derivations $\theta$ such that $\theta\left(I_{i}\right) \subset I_{i}^{m_{i}}$ for every $i$, where $I_{i}$ is the ideal of $p_{i}$. The module $D$ is graded and free of rank 2 ; the degrees of its homogeneous basis, called the exponents, form an important invariant of the divisor. We prove that under certain conditions on $\left(m_{i}\right)$ the exponents do not depend on $\left\{p_{i}\right\}$. Our main result asserts that if these conditions do not hold for $\left(m_{i}\right)$, then there exists a general position of $n$ points for which the exponents do not change. We give an explicit formula for them. We also exhibit some examples of degeneration of the exponents, in particular, those where the degeneration is defined by the vanishing of certain Schur functions. As an application and motivation, we show that our results imply Terao's conjecture (concerning the combinatorial nature of the freeness of hyperplane arrangements) for certain new classes of arrangements of lines in the complex projective plane.
\end{abstract}

\section{INTRODUCTION}

Arguably, the most intriguing conjecture in the theory of hyperplane arrangements is the Terao conjecture concerning the combinatorial character of the freeness of arrangements. The first interesting and open case is formed by 3 -arrangements, i.e., arrangements of projective lines in the complex projective plane $\mathbb{C P}^{2}$. Recent progress has been made by M. Yoshinaga (see Section 7) who found a new relation between the freeness of an arrangement $\mathcal{A}$ and its restriction $\mathcal{A}_{H}$ to an arbitrary hyperplane $H \in \mathcal{A}$. More precisely the hyperplanes of $\mathcal{A}_{H}$ have natural multiplicities, which allows one to consider the multi-arrangement $\tilde{\mathcal{A}}_{H}$. Then if $\mathcal{A}$ has rank $\ell+1$ and is free with the exponents $\left\{e_{0}=1, e_{1}, \ldots, e_{\ell}\right\}$ then $\tilde{\mathcal{A}}_{H}$ must be free with the exponents $\left\{e_{1}, \ldots, e_{\ell}\right\}$. For arrangements in $\mathbb{C P}^{2}$ this necessary condition is also sufficient (even if it is checked for only one line). This result brings to light multi-arrangements of points (i.e., effective divisors) on $\mathbb{C P}^{1}$.

In the rest of the paper we will consider multi-arrangements $\tilde{\mathcal{A}}=\left\{p_{1}, p_{2}, \ldots, p_{n}\right\}$ in $\mathbb{C P}^{1}$ with $n \geq 2$ and the positive integer multiplicity $m_{i}$ of $p_{i}(i=1,2, \ldots, n)$. We usually order the points subject to $m_{1} \geq m_{2} \geq m_{3} \geq \cdots \geq m_{n}$ and call $m=\left(m_{1}, m_{2}, \ldots, m_{n}\right)$ the multiplicity vector of $\tilde{\mathcal{A}}$. Also we put $\tilde{n}=\sum_{i=1}^{n} m_{i}$. If $m=(1,1, \ldots, 1)$, then $\tilde{\mathcal{A}}=\mathcal{A}$ and is called a simple arrangement.

Received by the editors July 25, 2005 and, in revised form, September 8, 2005.

2000 Mathematics Subject Classification. Primary 52C35, 14N20; Secondary 13N15, 15A36.

Research at MSRI was supported in part by NSF grant DMS-9810361.

(C)2007 American Mathematical Society Reverts to public domain 28 years from publication 
Choosing an appropriate coordinate system on $V=\mathbb{C}^{2}$ we can view the symmetric algebra $S=\mathcal{S}\left(V^{*}\right)$ as the polynomial algebra $\mathbb{C}[x, y]$ and fix linear forms $\alpha_{i}=x-z_{i} y \in S\left(z_{i} \in \mathbb{C}\right)$ such that $\operatorname{ker}\left(\alpha_{i}\right)=p_{i}$ for all $i$. Then the defining polynomial of $\tilde{\mathcal{A}}$ is $Q=\prod_{i=1}^{n}\left(x-z_{i} y\right)^{m_{i}}$.

The graded $S$-module of derivations

$$
D(\tilde{\mathcal{A}})=\left\{\theta \in \operatorname{Der}_{\mathbb{C}} S \mid \theta\left(\alpha_{i}\right) \in \alpha_{i}^{m_{i}} S\right\}
$$

is known to always be free (as a reflexive module over a ring of homological dimension 2) of rank 2, whence it is freely generated by two derivations whose degrees $e_{1}$ and $e_{2}$ are uniquely determined by $\tilde{\mathcal{A}}$. We always assume that $e_{1} \leq e_{2}$ and call the pair $\exp (\tilde{\mathcal{A}})=\left(e_{1}, e_{2}\right)$ the exponents of $\tilde{\mathcal{A}}$. As was noticed by G. Ziegler [7] (who first considered $D(\tilde{\mathcal{A}})$ ) the exponents are not in general determined by $m$, unlike in the case of a simple arrangement where $e_{1}=1$ and $e_{2}=n-1$ (see [2]).

The main result of this paper (Theorem 3.1) is that under certain conditions on the multiplicity vector $m$ there exists a general position set (more precisely, a nonempty set, open in the Zariski topology) of $n$ points on $\mathbb{C P}^{1}$ such that

$$
\exp (\tilde{\mathcal{A}})=\left(\left\lfloor\frac{1}{2} \sum_{i=1}^{n} m_{i}\right\rfloor,\left\lceil\frac{1}{2} \sum_{i=1}^{n} m_{i}\right\rceil\right)
$$

for every $n$-tuple of points in this position. The proof of the theorem is broken into several steps and occupies Sections 35. The main part of the proof is in Section 5 where we prove that a certain determinant is not identically zero as a polynomial in $z_{i}$ 's.

Also we study all the cases where $m$ does not satisfy these conditions. We prove that in these cases the exponents are determined by $m$ and give explicit formulas for them (Section 2). In Section 6, we consider examples of degeneration of the exponents, in particular, a case where the degeneration is defined by the vanishing of Schur functions for rectangular diagrams. Finally in Section 7 we recall the Terao conjecture and Yoshinaga theorem and exhibit several new classes of 3-arrangements for which our results imply the conjecture.

This paper was partially written when both authors were participating in the MSRI program on Hyperplane Arrangements and Applications. We are grateful to MSRI for support. We also are grateful to Hiro Terao and Masahiko Yoshinaga for useful discussions.

\section{Multiplicity VeCtors that Determine exponents}

First, for the convenience of the reader we recall Ziegler's generalization ([7], p. $351)$ of Saito's criterion ([2, Theorem 4.19) restricted to our case. Let $\theta_{1}, \theta_{2} \in$ $D(\tilde{\mathcal{A}})$ and in some coordinate system on $\mathbb{C P}^{1}$ we have $\theta_{i}=f_{i 1} \partial_{x}+f_{i 2} \partial_{y}$ for some homogeneous $f_{i j} \in S$ of the same degree, where $i, j=1,2$ and $\partial_{x}$ and $\partial_{y}$ are derivatives with respect to $x$ and $y$. Then $\left(\theta_{1}, \theta_{2}\right)$ is a basis of $D(\tilde{\mathcal{A}})$ if and only if the determinant $f_{11} f_{22}-f_{12} f_{21}$ is equal to $\tilde{Q}$ multiplied by a nonzero constant.

Now we mention two simple properties of the exponents $\left(e_{1}, e_{2}\right), e_{1} \leq e_{2}$, which we use frequently in the rest of the paper.

1. $e_{1}+e_{2}=\tilde{n}$.

This follows from the version of Saito's criterion of the freeness for multi-arrangements. 
2. Suppose $\mathcal{A}_{1} \subset \mathcal{A}_{2}$ are two multi-arrangements and $\tilde{n}\left(\mathcal{A}_{2}\right)=\tilde{n}\left(\mathcal{A}_{1}\right)+1$. If $\exp \left(\mathcal{A}_{1}\right)=\left(e_{1}, e_{2}\right)$ and $\exp \left(\mathcal{A}_{2}\right)=\left(e_{1}^{\prime}, e_{2}^{\prime}\right)$, then either $e_{1}^{\prime}=e_{1}\left(\right.$ whence $\left.e_{2}^{\prime}=e_{2}+1\right)$ or $e_{2}^{\prime}=e_{2}$ (whence $\left.e_{1}^{\prime}=e_{1}+1\right)$.

Indeed since $D\left(\mathcal{A}_{2}\right) \subset D\left(\mathcal{A}_{1}\right)$ we have $e_{i}^{\prime} \geq e_{i}, i=1,2$. Now the property follows from property 1 .

In the rest of the section we will consider several cases of multiplicity vectors $m$ where the exponents $\left(e_{1}, e_{2}\right)$ are uniquely determined by $m$ and do not depend on the position of the points. In most of these cases we will be able to exhibit a derivation of minimal degree from $D(\tilde{\mathcal{A}})$ as a function of $m$.

In the rest of the paper we always assume that a coordinate system is fixed on $\mathbb{C P}^{1}$ so that the defining polynomial of $\tilde{\mathcal{A}}$ is

$$
\tilde{Q}=x^{m_{1}} y^{m_{2}} \prod_{i=3}^{n}\left(x-z_{i} y\right)^{m_{i}}
$$

for some $z_{i} \in \mathbb{C} \backslash\{0\}$. Then any derivation from $D(\tilde{\mathcal{A}})$ has the form $f x^{m_{1}} \partial_{x}-g y^{m_{2}} \partial_{y}$ for some $f, g \in S\left(\partial_{x}\right.$ and $\partial_{y}$ are derivatives with respect to $x$ and $\left.y\right)$.

We can exhibit two derivations in $D(\tilde{\mathcal{A}})$ for future use. Put $\theta_{1}=\frac{\tilde{Q}}{x^{m_{1}}} \partial_{y}$. It is easy to see that $\theta_{1}$ is a homogeneous derivation having minimal degree among all the elements of $D(\tilde{\mathcal{A}})$ with $f=0$. Also put $\theta_{2}=\frac{\tilde{Q}}{Q} \theta_{E}$, where $\theta_{E}=x \partial_{x}+y \partial_{y}$ is the Euler derivation. Again it is easy to check that $\theta_{2}$ is a homogeneous derivation having minimal degree among all elements of $D(\tilde{\mathcal{A}})$ proportional to $\theta_{E}$.

Case 2.1. Let $m_{1} \geq \sum_{i=2}^{n} m_{i}$. Then $\theta_{1}$ is a derivation of minimal degree in $D(\tilde{\mathcal{A}})$. In particular, $\left(e_{1}, e_{2}\right)=\left(\sum_{i=2}^{n} m_{i}, m_{1}\right)$.

Proof. Since any homogeneous derivation $\theta$ from $D(\tilde{\mathcal{A}})$ with $f \neq 0$ satisfies $\operatorname{deg}(\theta) \geq$ $m_{1} \geq \sum_{i=2}^{n} m_{i}=\operatorname{deg}\left(\theta_{1}\right), \theta_{1}$ has the smallest degree in $D(\tilde{\mathcal{A}})$ and the statement follows.

Case 2.2. If $\tilde{n} \leq 2 n-2$, then $\theta_{2}$ is a derivation of minimal degree in $D(\tilde{\mathcal{A}})$. In particular,

$$
\left(e_{1}, e_{2}\right)=(\tilde{n}-n+1, n-1) .
$$

Proof. Notice $\operatorname{deg}\left(\theta_{2}\right)=\tilde{n}-n+1 \leq 2 n-2-n+1=n-1$. Suppose that there is $\theta \in D(\tilde{\mathcal{A}})$ such that $\operatorname{deg}(\theta)<\operatorname{deg}\left(\theta_{2}\right) \leq n-1$. Since $D(\tilde{\mathcal{A}}) \subset D(\mathcal{A})$ and the latter module is generated by $\theta_{E}$ together with a derivation of degree $n-1$ we have that $\theta=h \theta_{E}$ for a polynomial $h$. Applying $\theta$ to $\alpha_{i}$ we see that $h$ is divisible by $\frac{\tilde{Q}}{Q}$, which contradicts the condition on the degree of $\theta$.

Hence the degree of $\theta_{2}$ is minimal, and the result follows.

Remark 2.1. (i) Cases 2.1 and 2.2 meet at the case $m=(n-1,1, \ldots, 1)$. For this $m$ the derivations found in the cases give a minimal generating system of degrees $(n-1, n-1)$.

Indeed assume $\tilde{n} \leq 2 n-2$ and $m_{1} \geq \sum_{i=2}^{n} m_{i}$. Since every $m_{i} \geq 1$ we have $\sum_{i=2}^{n} m_{i}$ and $m_{1}$ both are not less than $n-1$, whence $\tilde{n}=2 n-2, m_{1}=n-1$ and $m_{i}=1$ for every $i>1$. 
(ii) The previous remark allows us to handle the case where $\tilde{n}=2 n-1$. Since $n \geq 2$, there exists an $m_{i}>1$. Decreasing this $m_{i}$ by 1 we obtain a new multiarrangement with exponents $(n-1, n-1)$. By property 2 , the exponents of the initial arrangement are $(n-1, n)$. In particular they are determined by $m$.

Together with Case 2.2, these comments imply that if the average of all $m_{i}$ is less than 2 , then the exponents are determined by $m$. If the average equals 2 , this is false. We however can give one example of $m$ for which it is true.

Example 2.2. If $m_{i}=2$ for all $1 \leq i \leq n$, then the exponents are $(n, n)$. Indeed we can exhibit two derivations of degree $n$ satisfying the Saito criterion.

Let an arrangement $\mathcal{A}$ be defined by $Q=x y \prod_{i=3}^{n}\left(x-z_{i} y\right)$ for some $n-2$ numbers $z_{i} \in \mathbb{C}$ and $\tilde{Q}=Q^{2}$. If $n=2$ we can take $\theta_{1}=x^{2} \partial_{x}$ and $\theta_{2}=y^{2} \partial_{y}$ and the result obviously follows.

Suppose $n>2$ and for every $i, 3 \leq i \leq n$, put $Q_{i}=\frac{Q}{x-z_{i} y}, h_{1}=\sum_{i=3}^{n} Q_{i}$, and $h_{2}=\sum_{i=3}^{n} z_{i} Q_{i}$. Then put

and

$$
\xi_{1}=h_{1} \theta_{E}+\frac{y}{x} Q \partial_{y}
$$

$$
\xi_{2}=h_{2} \theta_{E}-\frac{x}{y} Q \partial_{x}
$$

One can check straightforwardly that $\xi_{i} \in D(\tilde{\mathcal{A}})(i=1,2)$ and that the matrix of the coordinates of these derivations has determinant equal to $(n-2) Q^{2}$.

Let us remark that just a slightly different basis for this case was previously constructed in [3], Proposition 5.4.

If $n=2$ or 3 , then of course the exponents are determined by $m$ since there is only one position of 2 or 3 points on $\mathbb{C P}^{1}$ up to a projective isomorphism. For $n=4$, even for the average of the $m_{i}$ equal to 2 , the situation is different. It follows from the cases above that the vectors $(5,1,1,1),(4,2,1,1)$, and $(2,2,2,2)$ determine exponents uniquely (they are $(3,5)$ for the first vector and $(4,4)$ for the other two). On the other hand, for the vectors $(3,3,1,1)$ and $(3,2,2,1)$ the exponents depend on the position of 4 points on $\mathbb{C P}^{1}$ (see Section [6).

\section{THE MAIN THEOREM}

In this section, we are concerned with an arbitrary multiplicity vector $m$ not satisfying the strict inequalities studied in the previous section. Our main result asserts that $m$ uniquely determines the exponents $\left(e_{1}, e_{2}\right)$ for $n$ points in general position on $\mathbb{C P}^{1}$. (In this paper we understand by a general position set of $n$ points in $\mathbb{C P}^{1}$, a nonempty set in the configuration space $\left(\mathbb{C P}^{1}\right)^{n}$ open in the Zariski topology.) Roughly speaking these general position exponents are as close to being equal as possible. More precisely we are going to prove the following.

Theorem 3.1. Suppose $m$ has the following properties: $m_{1} \leq \sum_{i=2}^{n} m_{i}$ and $\tilde{n} \geq$ $2 n-2$. Then there exists a general position of $n$ points such that

$$
\exp (\tilde{\mathcal{A}})=\left(\left\lfloor\frac{1}{2} \tilde{n}\right\rfloor,\left\lceil\frac{1}{2} \tilde{n}\right\rceil\right)
$$


for every multi-arrangement $\tilde{\mathcal{A}}$ of points in this position having $m$ as the multiplicity vector.

The proof will be broken into several parts according to the following plan. First we focus on the case when $\tilde{n}$ is even. Then for $\tilde{n}$ odd the result can be deduced similarly to Remark 2.1 (i) (cf. section 5). For $\tilde{n}$ even the condition guaranteeing the existence of a derivation in $D(\tilde{\mathcal{A}})$ of degree less than $\tilde{n} / 2$ can be naturally expressed as the vanishing of the determinant of a square matrix $M$ of size $\sum_{i=3}^{n} m_{i}$ whose entries are monomials in $z_{i}, i=3,4, \ldots, n$. Thus the determinant of $M$ is a polynomial, $d$, in these indeterminates, and to prove the theorem it suffices to show that $d$ is not identically 0 . For this, in turn, it suffices to show that the leading monomial of $d$ (in some linear ordering of the monomials) has a nonzero coefficient.

Now the proof branches out. Since the set of rows of $M$ is by construction partitioned into blocks corresponding to the points $p_{3}, p_{4}, \ldots, p_{n}$, it is natural to use the Laplace formula for $d$. In most cases the coefficient of the leading term is the product of minors of $M$, one from each block of rows, and it is not hard to show that none of these minors vanishes. However, there are cases where the leading term is not a single product but a sum of several products of minors. This case is harder and requires a deeper analysis of the minors.

\section{The MAtrix $M$}

In this section, we assume that $\tilde{n}$ is even and put $e=\frac{1}{2} \tilde{n}-1$. We assume also that $m_{i} \geq 2$ for every $i$ and $m_{1}<\sum_{i=2}^{n} m_{i}$. Equivalently, $e-m_{1} \geq 0$, whence also $e-m_{2} \geq 0$. Any derivation $\theta \in D(\tilde{\mathcal{A}})$ of degree $e$ is of the form $\theta=x^{m_{1}} f(x, y) \partial_{x}-$ $y^{m_{2}} g(x, y) \partial_{y}$, where $f(x, y), g(x, y) \in S, \operatorname{deg}(f(x, y))=e-m_{1}$ and $\operatorname{deg}(g(x, y))=$ $e-m_{2}$. Write

$$
f(x, y)=\sum_{j=0}^{e-m_{1}} f_{j} x^{e-m_{1}-j} y^{j}
$$

and

$$
g(x, y)=\sum_{j=0}^{e-m_{2}} g_{j} x^{e-m_{2}-j} y^{j},
$$

where $f_{j}, g_{j} \in \mathbb{C}$. Besides since $\theta \in D(\tilde{\mathcal{A}})$, for all $i \geq 3$ we have $\theta\left(x-z_{i} y\right) \in$ $\left(x-z_{i} y\right)^{m_{i}} S$. Sacrificing homogeneity, we put $y=1$ and obtain the equivalent condition

$$
\left.\partial_{x}^{k}\left(\left.\theta\left(x-z_{i} y\right)\right|_{y=1}\right)\right|_{x=z_{i}}=0
$$

for all $0 \leq k \leq m_{i}-1$, where $\partial_{x}^{k}$ is the $k^{t h}$ derivative with respect to $x$. Treating the coefficients $f_{i}$ and $g_{j}$ as unknowns we consider $(*)$ as a system of equations for these unknowns. Explicitly the equation corresponding to the point $p_{i}(3 \leq i \leq n)$ and some $k\left(0 \leq k \leq m_{i}-1\right)$ is

$$
\sum_{j=0}^{e-m_{1}} f_{j} \frac{(e-j) !}{(e-j-k) !} z_{i}^{e-j-k}+z_{i} \sum_{j=0}^{e-m_{2}} g_{j} \frac{\left(e-m_{2}-j\right) !}{\left(e-m_{2}-j-k\right) !} z_{i}^{e-m_{2}-j-k}=0,
$$

where we agree that $z_{i}^{\ell}=0$ for $\ell<0$. 
The matrix $M$ is the matrix of coefficients of this system of equations which are polynomials in $z_{i}, i=3,4, \ldots, n$. A simple computation shows that the matrix is square; the number of rows as well as columns is $\sum_{3}^{n} m_{i}$. Moreover the set of rows of $M$ is partitioned into $n-2$ blocks $L_{3}, L_{4}, \ldots, L_{n}$, where the block $L_{i}$ does not contain $z_{j}$ with $j \neq i$. The set of columns of $M$ is partitioned into two blocks: the $f$-block and the $g$-block consisting of the coefficients at $f_{i}$ and $g_{j}$ respectively. The sizes of the blocks are $e-m_{1}+1$ and $e-m_{2}+1$ respectively. Notice that the $f$-block of the block $L_{i}$ is a Wronskian matrix of the functions $x^{e}, x^{e-1}, \ldots, x^{m_{1}}$ for their derivatives up to $m_{i}-1$ evaluated at $z_{i}$, i.e., an entry of the block has the form $\left.\partial_{x}^{k}\left(x^{e-\ell}\right)\right|_{x=z_{i}}, k=0,1, \ldots, m_{i}-1, \ell=0,1, \ldots, e-m_{1}$. The $g$-block of the block $L_{i}$ is a Wronskian matrix of the functions $x^{e-m_{2}}, x^{e-m_{2}-1}, \ldots, 1$ for the same range of derivatives as for the $f$-block, multiplied by $x$ and then evaluated at $z_{i}$. So its typical entry has the form $\left.\left(x \partial_{x}^{k}\left(x^{e-m_{2}-\ell}\right)\right)\right|_{x=z_{i}}, k=0,1, \ldots, m_{i}-1, \ell=0,1, \ldots, e-m_{2}$.

By construction of $M$ the vanishing of its determinant at some $(n-2)$-tuple $\left(z_{3}, \ldots, z_{n}\right)$ of pairwise different nonzero complex numbers is equivalent to the existence of a derivation of degree $e$ in $D(\tilde{\mathcal{A}})$, where $\tilde{\mathcal{A}}$ is the multi-arrangement defined by the formula (2.1). We can view $z_{i}$ as indeterminates and $\operatorname{det}(M)$ as a polynomial in these $n-2$ indeterminates. In the space $\mathbb{C}^{n-2}$ of all $(n-2)$-tuples of points the complement to the zero locus of $d$ is an open set in the Zariski topology. Thus Theorem 3.1 under the conditions on $m$ assumed in this section would follow from the following result.

Theorem 4.1. The polynomial d is not identically 0 .

The rest of the section is devoted to the proof of Theorem 4.1, which we break into several parts.

Lemma 4.2. The Wronskian of the power functions $x^{\lambda_{1}}, \ldots, x^{\lambda_{k}}$ where $\left(\lambda_{i}\right)$ is a strictly decreasing $k$-tuple of nonnegative integers is a monomial in $x$ of degree $\sum_{j=1}^{k} \lambda_{j}-\sum_{r=1}^{k-1} r$ with the coefficient

$$
(-1)^{\left\lfloor\frac{k}{2}\right\rfloor} \prod_{1 \leq i<j \leq k}\left(\lambda_{i}-\lambda_{j}\right)
$$

Proof. The first part of the statement follows immediately from the fact that every summand in the general formula for the determinant is the product of exactly one entry from each row and each column.

The second part of the statement can be proved by setting $x=1$ and transforming the obtained matrix to a Vandermonde matrix by applying row operations and induction on the number of rows. The sign $(-1)^{\left\lfloor\frac{k}{2}\right\rfloor}$ comes from the unusual ordering of the rows of the Vandermonde determinant.

In order to apply Lemma 4.2 we represent $d$ via a (repeated) Laplace's development corresponding to the fixed partition $\left(L_{3}, \ldots, L_{n}\right)$ of the set of rows of $M$. For that we need to consider ordered partitions $\beta=\left(B_{3}, \ldots, B_{n}\right)$ of columns such that $\left|B_{i}\right|=\left|L_{i}\right|=m_{i}$ for every $i$. Each $\beta$ defines a permutation $P(\beta)$ of all the columns of $M$. This gives a bijection of the set of these partitions with the set of permutations of the columns of $M$ such that the orderings of the columns inside 
subsequent intervals, $B_{i}$, of length $m_{i}$ are induced from $M$. Then we have

$$
d=\sum_{\beta} \operatorname{sign}(P(\beta)) \prod_{i=3}^{n} d\left(L_{i}, B_{i}\right),
$$

where the summation is over all the ordered partitions $\beta$ as above and $d\left(L_{i}, B_{i}\right)$ is the $m_{i} \times m_{i}$ minor of $M$ with rows from $L_{i}$ and columns from $B_{i}$.

Remark 4.3. The usual formula for signs of summands in Laplace's development differs from ours. Our formula can be easily checked by considering the signs of products of diagonal elements of each minor $d\left(L_{i}, B_{i}\right)$ (see [1]).

The degrees of the entries in each column are determined by the degree $r$ of the top element in the column; for brevity we say that the column has degree $r$.

In order to prove that the polynomial $d$ does not vanish identically it suffices to find a monomial of it with a nonzero coefficient. We will use for this purpose the leading term of $d$ in the lexicographic order generated by the reverse ordering of the indeterminates $z_{n}<z_{n-1}<\cdots<z_{3}$. The proof of Theorem 4.1 is immediate when the leading term is the product of (uniquely determined) minors, one from each block $L_{i}$. The simplest case when this happens is the case of 'no overlapping'.

Proposition 4.4. Suppose that the sets of degrees of (the top rows of) the columns in the $f$-block and those in the $g$-block are disjoint (equivalently, $m_{1}+m_{2}>\frac{\tilde{n}}{2}$ ). Then Theorem 4.1 holds.

Proof. Indeed in this case there are no two columns of the same degree. There is the unique partition $\beta$ of the columns such that the corresponding product of minors is the leading term of $d$; it is the partition corresponding to the identity permutation of the columns. The respective minors do not vanish by Lemma 4.2

In the rest of the proof we focus on the case where there is some overlapping. This means that several columns on the right flank of the $f$-block have the same degrees as several columns on the left flank of the $g$-block. The number of columns of this kind in the $f$-block is the same as this number for the $g$-block and equals $s=\frac{1}{2} \tilde{n}+1-\left(m_{1}+m_{2}\right)$. We denote by $O$ the set of these columns and denote its columns by $a_{1}, \ldots, a_{s}$ and $b_{1}, \ldots, b_{s}$, meaning that the degrees of $a_{i}$ and $b_{i}$ are the same $(i=1,2, \ldots, s)$ and $\operatorname{deg}\left(a_{i}\right)=\operatorname{deg}\left(a_{i+1}\right)+1(i=1,2, \ldots, s-1)$. Notice that $\operatorname{deg}\left(a_{1}\right)=\operatorname{deg}\left(b_{1}\right)=m_{1}+s-1=e-m_{2}+1$, and the number of columns with higher degrees is $e-\operatorname{deg}\left(a_{1}\right)=m_{2}-1$. The only minimal linearly dependent, over $S$, sets of columns in any block $L_{j}$ are $\left\{a_{i+1}, b_{i}\right\}(i=1,2, \ldots, s-1)$.

Even when overlapping occurs there still may be a unique choice of minors from the $L_{i}$ 's whose product equals the leading term of $d$. However, the simple partition that worked in the nonoverlapping case may not work now because some minors may vanish.

To analyze the situation deeper we need more notation. Consider a partition $\beta=\left(B_{3}, \ldots, B_{n}\right)$ of columns such that the corresponding product of minors equals the leading monomial of $d$ and the respective permutation $P(\beta)$. Then $P(\beta)$ is the identity permutation on the columns outside of $O$.

To express this in terms of partitions denote by $B_{i_{1}}$ and $B_{i_{2}}$ the first and the last blocks intersecting with $O$. Then the blocks $B_{3}, \ldots, B_{i_{1}-1}$ and $B_{i_{2}+1}, \ldots, B_{n}$ 
are determined uniquely as well as $r_{1}$ columns in $B_{i_{1}}$ and $r_{2}$ columns in $B_{i_{2}}$ not in $O$ (for some $0 \leq r_{j}<m_{i_{j}}, j=1,2$ ). For convenience, put $m_{i}^{\prime}=m_{i}$ if $i_{1}<i<i_{2}$ and $m_{i_{j}}^{\prime}=m_{i_{j}}-r_{j}$ for $j=1,2$. In particular, $\sum_{i=i_{1}}^{i_{2}} m_{i}^{\prime}=2 s$.

Now we can describe a (perhaps not unique) partition of $O$ recursively.

Proposition 4.5. Let $i_{1} \leq i \leq i_{2}$. Suppose $j$ is the minimal index such that the column $a_{j}$ is not chosen for all blocks $B_{i^{\prime}}$ with $i^{\prime}<i$ and $k$ is a similar index for $b_{k}$.

If $j \leq k$, then the block $B_{i}$ has the following columns from $O$ :

(1) $a_{j}, a_{j+1}, \ldots, a_{k-1}, a_{k}, b_{k}, b_{k+1}, \ldots, b_{m_{i}^{\prime}+j-2}$

(2) $a_{j}, a_{j+1}, \ldots, a_{m_{i}^{\prime}+j-1}$

(3) $a_{j}, a_{j+1}, \ldots, a_{k-1}, b_{k}$

(4) $a_{j}, a_{j+1}, \ldots, a_{k-1}, a_{k}$

If $j>k$, then $m_{i}^{\prime}=2, j=k+1$ and the columns in $B_{i} \cap O$ are if $m_{i}^{\prime} \geq k-j+2$,

if $m_{i}^{\prime} \leq k-j$,

if $m_{i}^{\prime}=k-j+1$,

if $m_{i}^{\prime}=k-j+1$

and either $i=i_{2}$ or $m_{i+1}^{\prime} \leq 2$.

$b_{k}, b_{j}$.

Proof. We use induction on $i$. It is obvious for $i=i_{1}$ and follows from the inductive hypothesis for $i>i_{1}$ that the indexes of columns $a_{t}$ and $b_{t}$ available for $B_{i}$ form the intervals $j \leq t \leq s$ and $k \leq t \leq s$ respectively. Choosing the columns of maximal available degrees and avoiding linearly dependent pairs, we immediately obtain the first three cases for $j \leq k$.

The fourth case is more subtle. If $m_{i}^{\prime}=k-j+1$, then the columns from $a_{j}$ to $a_{k}$ have the maximal degrees and are independent, whence seem to be appropriate for $B_{i}$. Assume however that $i \neq i_{2}$ and $m_{i+1}^{\prime} \geq 3$. Then with the above choice of columns for $B_{i}$ the block $B_{i+1}$ has columns $b_{k}, b_{k+1}, b_{k+2}, \ldots$. However the choice of columns for $B_{i}$ from the third case gives the same degree minor in $L_{i}$ and allows the higher degree choice $a_{k}, a_{k+1}, b_{k+1}, \ldots$ for a minor in $L_{i+1}$. Thus, the choice (4) in this case does not work. In the case where $m_{i+1}^{\prime}=1$ or 2 , this obstruction disappears and both choices (3) and (4) for $B_{i}$ give nonzero minors of the same degree.

Finally consider the case $j>k$. This condition means that the column $a_{k}$ was used in the previous blocks and $b_{k}$ was not. Applying the inductive hypothesis we can see that it is possible if and only if the choice (4) occurs in $L_{i-1}$ whence $m_{i}^{\prime}=2=k-j+1$. The choice of columns for $B_{i}$ is now clear.

Remark 4.6. Proposition 4.5 can be used to show that even if $O \neq \emptyset$, there may be just one product of minors equal to the leading term of $d$. Moreover, it is possible in general to compute the index $r\left(i_{1} \leq r \leq i_{2}\right)$ of the block, where the nonuniqueness starts, directly from the vector $m$. Since we won't use this in the paper we omit this computation.

We will need however some properties of $r$, which we collect in the following corollary, which follows immediately from Proposition 4.5 , 
Corollary 4.7. Suppose that for a multiplicity vector $m$ there are at least two different partitions of $O$ whose corresponding products of minors equal the leading monomial of $d$. Then

(1) The union of $\bigcup_{r^{\prime}<r} B_{r^{\prime}}$ and the first $m_{r}-1$ columns of $B_{r}$ consist of all the columns of degree larger than $\operatorname{deg}\left(a_{t}\right)$ for some $t \leq s$ satisfying the equality

$$
2(t-1)+m_{2}=\sum_{i=3}^{r} m_{i} .
$$

(2) Either $t=s$ or $m_{i}=2$ for all $i>r$.

(3) The set of columns $O^{\prime}=\left\{a_{t}, a_{t+1}, \ldots, a_{s}, b_{t}, b_{t+1}, \ldots, b_{s}\right\}$ is partitioned by the blocks from $B_{r}$ to $B_{i_{2}}$. This partition has the following properties:

(a) the block $B_{r}$ has only one column from $O^{\prime}$, either $a_{t}$ or $b_{t}$ (which implies that $B_{i_{2}}$ has either $a_{s}$ or $b_{s}$ and no other columns from $O$ );

(b) if the second column of a block is $a_{j}$, then the first column of the next block is $b_{j}$ and vice versa;

(c) if the first column of a block is $a_{j}$, then its second column is either $a_{j+1}$ or $b_{j+1}$;

(d) if the first column of a block is $b_{j}$, then its second column is $b_{j+1}$.

The ordered partitions of $O^{\prime}$ having the properties of the previous corollary are in one-to-one correspondence with some permutations of $O^{\prime}$ that we call admissible.

Example 4.8. Using the notation of the previous corollary, if $k=1$ and $s=2$, then there exist the following 3 admissible partitions of $O=O^{\prime}$ :

$$
a_{1}\left|b_{1} b_{2}\right| a_{2}, b_{1}\left|a_{1} a_{2}\right| b_{2}, b_{1}\left|a_{1} b_{2}\right| a_{2} .
$$

It is easy to explicitly compute the minors involved in Corollary 4.7. Under the conditions of Proposition 4.5 consider any product of minors giving a maximal degree monomial of $d$. Recall that the minor $\mu_{i}=d\left(L_{i}, B_{i}\right)$ is a monomial in $z_{i}$ with some coefficient $c_{i} \in \mathbb{Z}, i=3, \ldots, n$.

Lemma 4.9. The coefficients, $c_{i}$, are as follows.

If $i_{2}-r>1$, then

(i) $c_{i}=-1$ for $r<i \leq i_{2}$ if both columns of $\mu_{i}$ are from the $f$-block or both columns are from the $g$-block, and $c_{i}=-2$ if the columns are from different blocks.

If $i_{2}=r+1$, then

(ii) $c_{i_{2}}=(-1)^{\left\lfloor\frac{m_{i_{2}}}{2}\right\rfloor} \pi\left(m_{i_{2}}-1\right)$ ! if the first column of $\mu_{i_{2}}$ is $b_{s}$, and $c_{i_{2}}=$ $(-1)^{\left\lfloor\frac{m_{i_{2}}}{2}\right\rfloor} \pi m_{i_{2}}$ ! if the first column is $a_{s}$, where $\pi=\prod_{j=1}^{m_{i_{2}}-2} j$ ! for $m_{i_{2}}>2$ and $\pi=1$ otherwise.

For arbitrary $i_{2}-r \geq 1$,

(iii) $c_{r}=(-1)^{\left\lfloor\frac{m_{r}}{2}\right\rfloor} \pi^{\prime}\left(m_{r}-1\right)$ ! if the last column of $\mu_{r}$ is $a_{k}$, and $c_{r}=$ $(-1)^{\left\lfloor\frac{m_{r}}{2}\right\rfloor} \pi^{\prime} m_{r}$ ! if the last column is $b_{k}$, where $\pi^{\prime}=\prod_{j=1}^{m_{r}-2} j !$ for $m_{r}>2$ and $\pi^{\prime}=1$ otherwise.

This lemma follows immediately from Corollary 4.7 and Lemma 4.2

Now we are ready to prove that even when the leading monomial of the determinant $d$ is not just a product of minors but the sum of several of those, its coefficient does not vanish. We prove this by computing that coefficient. 
For each admissible permutation $P$ of $O^{\prime}=\left(a_{k}, \ldots, a_{s}, b_{k}, \ldots, b_{s}\right)$ (i.e., a permutation that defines a partition satisfying Corollary 4.7) consider the product $\prod(P)=\prod c_{i}$, where $r \leq i \leq i_{2}$ and $c_{i}$ is defined for the block $B_{i}$ in Lemma 4.9. Notice that the product $\pi^{\prime}\left(m_{r}-1\right)$ ! can be factored out of $\prod(P)$ for each $P$ along with the product $\prod_{i=3}^{r-1} c_{i} \prod_{i=i_{2}+1}^{n} c_{i}$. More precisely for an arbitrary admissible permutation $P$ put

$$
\prod(P)=\left((-1)^{\left\lfloor\frac{m_{r}}{2}\right\rfloor+\left\lfloor\frac{m_{i_{2}}}{2}\right\rfloor} \pi^{\prime}\left(m_{r}-1\right) \prod_{i=3}^{r-1} c_{i} \prod_{i=i_{2}+1}^{n} c_{i}\right) C(P)
$$

for some $C(P) \in \mathbb{C}$.

Proposition 4.10. If $i_{2}=r+1$, then

$$
\sum_{P} \operatorname{sign}(P) C(P)=\pi\left(m_{i_{2}}-1\right) !\left(1-m_{r} m_{i_{2}}\right) ;
$$

if $i_{2}>r+1$, then

$$
\sum_{P} \operatorname{sign}(P) C(P)=(-1)^{\left\lceil\frac{u+2}{2}\right\rceil}\left(\left(m_{r}-1\right) u+1\right),
$$

where the sum is taken over all the partitions $P$ of $O^{\prime}$ satisfying Corollary 4.7 and $u=i_{2}-r+1$.

Proof. (i) Suppose $i_{2}=r+1$. Lemma 4.9 (ii) and (iii) imply that there are only two admissible permutations. The formula (4.1) follows also from that lemma.

(ii) Suppose that $i_{2}>r+1$, i.e., $u>2$. Corollary 4.7 implies that $m_{i_{2}}=2$, whence the left-hand side of (4.2) depends only on $m_{r}$ and $u$. We denote it by $\sigma\left(m_{r}, u\right)$ and put $\sigma(u)=\sigma(2, u)$.

Let us consider first the most important case where $m_{r}=2$. The key observation here is the following recursive formula:

$$
\sigma(u)=\sigma(u-2)+(-1)^{u} 2 \sigma(u-1)
$$

for every $u \geq 4$. Consider first all permutations $P$ with $a_{k}$ as the first element. Then the beginning of $P$ is $a_{k}\left|b_{k} b_{k+1}\right| a_{k+1}$. That shows that $C(P)=C\left(P^{\prime}\right)$, where $P^{\prime}$ is the restriction of $P$ to the rest of $O^{\prime}$. Observe that the size of the set is $u-2$ and $\operatorname{sign}\left(P^{\prime}\right)=\operatorname{sign}(P)$. This explains the first summand of the formula.

Then consider all permutations $P$ with $b_{k}$ as the first element. This defines the beginning of $P$ as $b_{k} \mid a_{k}$. Thus $C(P)=(-2) C\left(P^{\prime}\right)$, where $P^{\prime}$ is again the restriction of $P$ to the rest of $O^{\prime}$, this time of size $u-1$. Besides, $\operatorname{sign}\left(P^{\prime}\right)=(-1)^{u-1} \operatorname{sign}(P)$, as is easy to see by counting transpositions. That explains the second summand.

Augment the recursive formula by the initial conditions $\sigma(2)=3$ and $\sigma(3)=-4$ (for $u=3$ there are 3 admissible orderings giving the summands equal to $2,2,-8$, cf. Example 4.8). Using the recursive formula and the initial conditions, formula (4.2) for $m_{r}=2$ can be proved by induction on $u$.

If $m_{r}>2$, then the recursive formula is a little different:

$$
\sigma\left(m_{r}, u\right)=\sigma(u-2)+(-1)^{u} m_{r} \sigma(u-1),
$$

although the proof is the same. Substituting (4.2) for $m_{r}=2$ in this formula we complete the proof.

Proposition 4.10 completes the proof of Theorem 4.1 . 


\section{Proof of Theorem 3.1}

In this section we finish the proof of Theorem 3.1

Recall that we consider multi-arrangements $\tilde{\mathcal{A}}$ with the following conditions on the multiplicity vector $\left(m_{1}, m_{2}, \ldots, m_{n}\right)$ such that $m_{1} \geq m_{2} \geq \cdots \geq m_{n}$ :

(i) $m_{1} \leq \sum_{i=2}^{n} m_{i}$;

(ii) $\tilde{n}=\sum_{i=1}^{n} m_{i} \geq 2 n-2$.

If $\tilde{n}$ is even, then condition (i) allows us to write a square matrix $M$ whose determinant $d=\operatorname{det} M$ is a polynomial in the coordinates of the points of $\mathcal{A}$. The equation $d=0$ describes the locus in $\left(\mathbb{C P}^{1}\right)^{n-2}$ of all arrangements $\mathcal{A}$ such that $e_{1}(\tilde{\mathcal{A}})<\frac{n}{2}$. For the case $m_{n}>1$ (i.e., $m_{i}>1$ for every $i$ ), we proved in the previous section that $d$ is not identically 0 , which implies the statement of the theorem for this case.

Now we consider the general case where the multiplicity vector is arbitrary (satisfying (i) and (ii)). We apply induction on the number, $\alpha$, of multiplicities equal to 1 using as the base, results of the previous section for $\alpha=0$. Suppose $\alpha>0$ (in particular $m_{n}=1$ ) and consider $m^{\prime}=\left(m_{1}, \ldots, m_{n-1}\right)$. If $\tilde{n}$ is odd, then the result follows immediately from the inductive hypothesis for $m^{\prime}$, the monotonicity of $e_{1}$, and the fact that the pre-image of a general position set under a coordinate projection is in general position. Thus we can assume that $\tilde{n}$ is even.

Assume that the result does not hold for $m$. Since $\tilde{n}$ is even we can again bring the matrix $M$ into consideration. The assumption implies that $d$ is the 0 polynomial, i.e., every arrangement with the multiplicity vector $m$ has

$$
e_{1} \leq \frac{\tilde{n}}{2}-1
$$

In particular if we fix a multi-arrangement $\tilde{\mathcal{A}}^{\prime}$ of $n-1$ points in general position with the multiplicity vector $m^{\prime}$, then adjoining any extra point $q$ (with multiplicity 1 ) we obtain a multi-arrangement satisfying (5.1). Applying the inductive hypothesis to $m^{\prime}$ we obtain that for $\tilde{\mathcal{A}}^{\prime}$ we have $\exp \left(\tilde{\mathcal{A}}^{\prime}\right)=\left(\frac{\tilde{n}}{2}-1, \frac{\tilde{n}}{2}\right)$. If $\theta$ is a (unique up to a nonzero multiplicative constant) derivation from $D\left(\tilde{\mathcal{A}}^{\prime}\right)$ of degree $\frac{\tilde{n}}{2}-1$, then for any point, $q, \theta$ will preserve $q$. Thus, $\theta$ is in $D(\mathcal{B})$, where $\mathcal{B}$ is an arbitrary (simple) arrangement. Choose $\mathcal{B}$ of a cardinality greater than $\frac{\tilde{n}}{2}+1$. Then $\exp (\mathcal{B})=(1, e)$, where the exponent 1 corresponds to the Euler derivation $\theta_{E}$ and $e>\frac{\tilde{n}}{2}$. Hence, $\theta$ is proportional to $\theta_{E}$, i.e., $\theta=P \theta_{E}$ for a homogeneous polynomial $P$, since besides $\theta \in D(\tilde{\mathcal{A}})$ we have $\operatorname{deg}(P) \geq \tilde{n}-n$ (see Section 2), which implies

$$
\tilde{n}-n+1 \leq \frac{\tilde{n}}{2}-1 \text {. }
$$

The inequality (5.2) is equivalent to $\tilde{n} \leq 2 n-4$, which contradicts condition (ii) of the theorem. This contradiction completes the proof.

\section{Degeneration of the exponents}

In this section, we consider several cases of degeneration of exponents, i.e., examples of (classes of) multi-arrangements satisfying the conditions of Theorem 3.1 and having $e_{1}<\frac{\tilde{n}}{2}$. We assume for convenience that $\tilde{n}$ is even and as before represent 
the defining polynomial of a multi-arrangement as

$$
\tilde{Q}=x^{m_{1}} y^{m_{2}} \prod_{i=3}^{n}\left(x-z_{i} y\right)^{m_{i}}
$$

$\left(m_{i} \leq m_{j}\right.$ for $\left.j<i\right)$. It is necessary for degeneration that the $(n-2)$-tuple $\left(z_{3}, \ldots, z_{n}\right)$ annihilates the polynomial $d$.

Remark 6.1. The polynomial $d$ has a factor whose zero locus does not contain acceptable $(n-2)$-tuples. Factoring $z_{i}$ from each row of a block $L_{i}$ of the matrix $M$, then subtracting similar rows of different blocks and factoring differences $\left(z_{i}-z_{j}\right)$ we obtain

$$
d=\prod_{k=3}^{n} z_{k}^{m_{k}} \prod_{3 \leq j<i \leq n}\left(z_{j}-z_{i}\right)^{m_{i}} d_{1}
$$

for some polynomial $d_{1}$.

We conjecture that $d_{1}$ is not divisible by any $z_{i}$ or $z_{i}-z_{j}$. There could be however $(n-2)$-tuples in the zero locus of $d_{1}$ that do not define an arrangement (with $n$ points). For instance, if $m=(6,4,3,2,1)$, then $d_{1}(1,1,1)=0$.

\section{Examples.}

1. $m=(3,3,1,1)$. This example was considered first by G. Ziegler [7]. An arbitrary multi-arrangement with the multiplicity vector $m$ is projectively equivalent to $x^{3} y^{3}(x-y)(x-z y)(z \in \mathbb{C} \backslash\{0,1\})$ and the matrix $M$ is

$$
M=\left(\begin{array}{cc}
1 & -1 \\
z^{3} & -z
\end{array}\right)
$$

The polynomial $d_{1}=z+1$ vanishes at $z=-1$. Thus the only degenerate case is $x^{3} y^{3}\left(x^{2}-y^{2}\right)$, which has exponents $(3,5)$. Notice that the degeneration here happens exactly in the case where the quadruple $\left(p_{1}, p_{2}, p_{3}, p_{4}\right)$ of points is harmonic (i.e., its cross-ratio equals -1 ).

2. An interesting generalization of the previous example is given by the vector $m=(k, k, 1, \ldots, 1)$, where $k \geq 3$ and $n=k+1$. A corresponding multiarrangement can be written as $x^{k} y^{k} \prod_{i=1}^{k-1}\left(x-z_{i} y\right)$. As a straightforward computation shows, this arrangement has $e_{1}=k$ (the minimal possible) if and only if the set $\left\{z_{1}, z_{2}, \ldots, z_{k-1}\right\}$ is equal to the set of all roots of degree $(k-1)$ of a nonzero (complex) number. Notice that $\tilde{n}=3 k-1$, whence $m$ satisfies the conditions of Theorem 3.1 Thus, this series of examples shows that the value of $e_{1}$ can decrease with respect to the general position by any positive integer.

3. Let us generalize the previous example further. Put $m=\left(m_{1}, m_{2}, 1, \ldots, 1\right)$ with an arbitrary $n$ satisfying $m_{1}-m_{2}<n-2<m_{1}+m_{2}$. (We still assume that $\tilde{n}=m_{1}+m_{2}+n-2$ is even, which is not very important.) It is easy to check that the conditions on $m_{1}, m_{2}$ and $n$ imply the conditions (i) and (ii) of Theorem 3.1 whence for an arrangement in general position $e_{1}=\frac{\tilde{n}}{2}$. The matrix $M$ for this example differs from a Vandermonde matrix by only one gap in the degrees, down from $m_{1}-1$ to $\frac{m_{1}-m_{2}+n-4}{2}$ (after factoring out $z_{i}$ from the $i$ th row for every 
i). In other words $d_{1}= \pm s_{\lambda}$, where $s_{\lambda}$ is the Schur function for the rectangular partition diagram with base $\frac{m_{1}+m_{2}-n}{2}$ and height $\frac{-m_{1}+m_{2}+n-2}{2}$. Thus we obtain a description of the set of arrangements with degenerate $e_{1}$ as the zero locus of the special 'rectangular' Schur function.

4. Let us recall Example 1 in this section. It has $\tilde{n}=8$, which is the smallest value of $\tilde{n}$ that can allow different $e_{1}$. Besides Example 1, there is only one more vector $m$ with $\tilde{n}=8$ such that $e_{1}$ is not determined by $m$. It is $m=(3,2,2,1)$. Indeed $m$ satisfies the conditions (i) and (ii) of Theorem 3.1 and for an arrangement $x^{3} y^{2}\left(x-z_{1} y\right)^{2}\left(x-z_{2} y\right)$ the condition for $e_{1}=3$ is $d_{1}=-2 z_{1}+z_{2}=0$. This defines one (up to projective isomorphism) arrangement with $z_{1}=1$ and $z_{2}=2$. Notice that again the quadruple $(0,-2, \infty,-1)$ of points in $\mathbb{C P}^{1}$ is harmonic (cf. the order of points from Example 1).

One more class of examples with a similar flavor is given by Proposition 7.4.

\section{The Terao conjecture}

As we mentioned in the Introduction the main motivation for this work is the Terao conjecture and Yoshinaga theorem for 3-arrangements.

Conjecture 7.1 (Terao, 2]). If two hyperplane arrangements over the same field have isomorphic intersection lattices and one of them is free, then the other is free as well.

This conjecture was posed (as a question) more than 20 years ago (4]) and is still open. The first nontrivial (and already hard) case is formed by arrangements in $\mathbb{C}^{3}$ or equivalently in $\mathbb{C P}^{2}$. The main evidence in favor of the conjecture is a theorem by Terao ([2], Theorem 4.61) that for a free arrangement its exponents are precisely the roots of the characteristic polynomial of its intersection lattice, whence are determined by the intersection lattice. We also mention a result from ([6]) that in the space of all arrangements with a fixed intersection lattice the free ones form a set open in the Zariski topology.

Some recent progress has been made by Yoshinaga in [5]. We paraphrase here the relevant part of his Theorem 2.2.

Theorem 7.2 (Yoshinaga, [5]). If an arrangement $\mathcal{A}$ of lines in $\mathbb{C P}^{2}$ is free with exponents $1, e_{1}, e_{2}$, then its restriction to every $\ell \in \mathcal{A}$ has (as a multi-arrangement) exponents $e_{1}, e_{2}$. If the characteristic polynomial of $\mathcal{A}$ has roots $1, d_{1}, d_{2}$ and the restriction of $\mathcal{A}$ to some line has exponents $d_{1}, d_{2}$, then $\mathcal{A}$ is free.

Yoshinaga's theorem implies in particular that if an arrangement $\mathcal{A}$ in $\mathbb{C P}^{2}$ gives a counterexample to the Terao conjecture, then its restrictions to all lines allow simultaneous degeneration of their minimal exponents. Since the multiplicity vectors of the restrictions are determined by the intersection lattice of $\mathcal{A}$, any time the multiplicity vector on one of the lines determines the exponents we obtain a class of arrangements (or lattices) satisfying the conjecture.

Let us give some examples to this effect. To avoid any confusion let us emphasize that the multiplicity $\bar{m}(p)$ of a point $p$ for an arrangement $\mathcal{A}$ in $\mathbb{C P}^{2}$ is the number of all lines of the arrangement passing through $p$. If $p \in \ell \in \mathcal{A}$, then the multiplicity of $p$ in the restricted to $\ell$ multi-arrangement is $m(p)=\bar{m}(p)-1$. 
Terao's conjecture holds for the following classes of arrangements $\mathcal{A}$ in $\mathbb{C P}^{2}$.

1. There exists a line in $\mathcal{A}$ whose intersections with other lines of $\mathcal{A}$ are concentrated at no more than 3 points.

2. There exists a line in $\mathcal{A}$ such that all points on it have multiplicities not larger than 3.

3. There exists a line in $\mathcal{A}$ such that the multiplicity vector of the restricted multi-arrangement satisfies $m_{1} \geq \sum_{i=2}^{n} m_{i}$.

4. There exists a line in $\mathcal{A}$ such that the average of the multiplicities of the restricted multi-arrangement is less than 2 .

Remark 7.3. If $\mathcal{A}$ is a line arrangement in $\mathbb{C P}^{2}$ such that $|\mathcal{A}| \leq 8$, then either condition 1 or 4 holds. Hence, for $|\mathcal{A}| \leq 8$ Terao's conjecture holds.

In fact we can prove that a larger class of arrangements satisfies Terao's conjecture.

Proposition 7.4. Let $\mathcal{A}$ be a line arrangement in $\mathbb{C P}^{2}$. If there exists an intersection point $p$ such that $\bar{m}(p)>\frac{1}{2}(|\mathcal{A}|-3)$, then Terao's conjecture holds for $\mathcal{A}$ (i.e. for all arrangements with the intersection lattice isomorphic to that of $\mathcal{A}$ ).

Proof. Suppose $p$ satisfies the condition and denote by $\ell_{1}, \ell_{2}, \ldots, \ell_{k}$ all the lines of $\mathcal{A}$ passing through $p$, where $k=\bar{m}(p)$. There are two alternatives.

(i) There are two lines $\ell, \ell^{\prime} \in \mathcal{A}$ such that $p^{\prime}=\ell \cap \ell^{\prime} \notin \ell_{i}$ for every $i$. Then the points $\ell \cap \ell_{1}, \ldots, \ell \cap \ell_{k}, p^{\prime}$ are pairwise distinct. This implies that the number $k^{\prime}$ of intersection points on $\ell$ satisfies $k^{\prime} \geq k+1>\frac{1}{2}(|\mathcal{A}|-1)$, whence the average of the multiplicities of the restriction of $\mathcal{A}$ to $\ell$ is less than 2. The result follows from (4) above.

(ii) Every point of intersection lies on a line passing through $p$. This implies that the intersection lattice of $\mathcal{A}$ is supersolvable (see 2, pp. 30, 31). Since every arrangement with a supersolvable lattice is free ([2, Theorem 4.58) the result follows.

In particular, Remark 7.3 generalizes to the following.

Corollary 7.5. Terao's conjecture holds for line arrangements in $\mathbb{C P}^{2}$ of cardinality less than 11.

Proof. Indeed every arrangement with at most 10 lines either satisfies the condition 2 above or the condition of the previous proposition.

\section{REFERENCES}

1. T. Muir, A treatise on the theory of determinants, Revised and enlarged by William H. Metzler, Dover Publications, Inc., New York, 1960, vii+766 pp. MR0114826 (22:5644)

2. P. Orlik and H. Terao, Arrangements of hyperplanes, Springer Verlag, 1992. MR.1217488 (94e:52014)

3. L. Solomon and H. Terao, The double Coxeter arrangement, Commentarii Math. Helvetici 73 (1998), no. 2, 237-258. MR1611699(99c:52018)

4. H. Terao, The exponents of a free hypersurface, Singularities, editor P. Orlik, Proc. Symp. Pure. Math 40, Pt. 2 (1983), 561-566. MR0713280(85a:32012)

5. M. Yoshinaga, Characterization of a free arrangement and conjecture of Edelman and Reiner, Invent. Math. 157 (2004), no. 2, 449-454. MR2077250(2005d:52044) 
6. S. Yuzvinsky, Free and locally free arrangements with a given intersection lattice, Proc. of AMS 118 (1993), 745-752. MR 1160307 (93i:52022)

7. G. Ziegler, Multi-arrangements of hyperplanes and their freeness, in Singularities, Contemp. Math. 90 (1986), 345-359. MR1000610(90e:32015)

Department of Mathematics, University of Oregon, Eugene, Oregon 97403

E-mail address: mwakefie@math.uoregon.edu

Current address: Department of Mathematics, Hokkaido University, Kita 10, Nishi 8, Kita-Ku, Sapporo, Hokkaido 060-0810, Japan

Department of Mathematics, University of Oregon, Eugene, Oregon 97403

E-mail address: yuz@math.uoregon.edu 